\title{
Grain Structure and Microhardness Development of Twin-Roll Cast Aluminum Strips after Constrained Groove Pressing
}

\author{
J. Bajer ${ }^{a, *}$, M. Cieslar ${ }^{a}$, M. Šlapáková ${ }^{a}$, M. Zimina $^{b}$, Z. NovÝ ${ }^{c}$ And A. Materna ${ }^{d}$ \\ ${ }^{a}$ Charles University, Faculty of Mathematics and Physics, Ke Karlovu 5, Prague 2, 121 16, Czech Republic \\ ${ }^{b}$ Research Center Rez, Hlavní 130, Husinec-Řžz, 250 68, Czech Republic \\ ${ }^{c}$ COMTES FHT, Průmyslová 995, Dobřany, 334 41, Czech Republic \\ ${ }^{d}$ Czech Technical University in Prague, Faculty of Nuclear Sciences and Physical Engineering, \\ Trojanova 13, Prague 2, 120 00, Czech Republic
}

\begin{abstract}
Twin-roll cast aluminum strips based on EN AW 3003 alloy with a small addition of Zr and Cr further processed by constrained groove pressing (CGP) were studied in this work. Vickers microhardness measurements were used to investigate an inhomogeneous distribution of microhardness as a result of an inhomogeneous strain distribution imposed by CGP. Results are consistent with the finite element method simulations of the total equivalent plastic strain which predicts higher accumulated strain in areas close to the edges of CGP dies. Microhardness saturates after two CGP cycles due to a dynamic recovery. Electron backscatter diffraction, light optical microscopy and transmission electron microscopy were used to study grain and dislocation structure and to compare domains with higher and lower microhardness. The sample after 3 CGP cycles exhibits an unusual mechanical behavior. Domains with higher microhardness exhibit a higher degree of recovery with a lower dislocation density than domains with lower microhardness.
\end{abstract}

DOI: 10.12693/APhysPolA.134.909

PACS/topics: 61.72.Ff, 68.35.bd, 68.37.Lp, 81.05.Zx, 81.40.--Z

\section{Introduction}

Combining of unconventional materials and new casting and forming methods could lower financial costs and environmental burden in the sheet producing industry. Therefore, established technologies have to be modified in order to fulfill the demands on formability, hardness, high temperature resistance and other characteristics of the material. The twin-roll casting (TRC) minimizes a number of processing steps which allows production of sheets and strips with required thickness, partially omitting several steps such as cropping, additional rolling or annealing. High cooling rates during TRC lead to grain refinement, formation of finely dispersed primary particles and solid solution with high supersaturation [1]. On the other hand, inhomogeneity, such as band structure and central segregations, can be found in TRC strips [2]. TRC strips cast to the final thickness therefore have to be subjected to several enhanced processing steps if their potential should be fully exploited. These include heat treatment, severe plastic deformation (SPD) or combination of both.

SPD-processed metals usually contain finer grains and high amount of dislocations as a consequence of undergoing plastic deformation [3]. Increase of strength comes from both grain size-related strengthening (proportional to an inverse square root of the grain size through HallPetch relation) [4] and dislocation-related strengthening

*corresponding author; e-mail: JanBajer69@seznam.cz (proportional to a square root of the dislocation density) [5]. Results in an agreement with these relations were obtained e.g. by Tsuji et al. [6]. They performed tensile tests on accumulative roll-bonding (ARB) aluminum and interstitial-free steel having grain sizes ranging from $20 \mu \mathrm{m}$ to $0.2 \mu \mathrm{m}$, and found that grain refinement by SPD led to tensile strength 2.7-3.4 times larger than that of the initial materials.

On the other hand, some experiments performed on materials with fine grains brought results which differ from the Hall-Petch relation. Kamikawa et al. [7] investigated a relationship between the yield strength and the grain size of pure aluminum produced by ARB and annealing. They showed that the variation of the yield stress with respect to the grain size significantly deviated from the conventional Hall-Petch relation for the grain size smaller than $10 \mu \mathrm{m}$. Gubicza et al. [8] showed that the yield strength of metals $(\mathrm{Al}, \mathrm{Al}-\mathrm{Mg}$ alloys, $\mathrm{Cu}$ and $\mathrm{Ni}$ ) processed by equal channel angular pressing (ECAP) could be described solely by the dislocationrelated strengthening using the Taylor equation without consideration of the grain size effect, as a first approximation. Thus, for SPD-processed metals, the degree of the grain size-related strengthening relative to the observed yield strength has not been fully understood [3].

Moreover, UFG materials sometimes show unexpected mechanical properties. It was reported that their strength could increase by annealing and decrease by predeformation which was called "hardening by annealing and softening by deformation" [6, 7, 9-12]. These phenomena are entirely opposite to the typical behavior of materials with the grain size lager than several tens of mi- 
crometers [9]. Experiments performed on TRC AA3003 aluminum alloy subjected to ECAP also reported this anomalous behavior [10].

It can be explained by the correlation between the strength and the density of dislocation sources in the nanostructured metals. Firstly, dislocations are activated and multiplied with difficulty within the annealed ultrafine grains due to their small size and low density of dislocation sources. In the SPD processed materials or predeformed ones, however, free dislocations as well as dislocation sources remain in the microstructure, resulting in relatively easier yielding $[6,7,9]$.

This work is focused on constrained groove pressing (CGP) which seems to be one of the most promising SPD methods producing strip and plane materials. Presence of the phenomenon of softening by deformation or dynamic recovery was investigated on EN AA3003 aluminum alloy with small additions of $\mathrm{Zr}$ and $\mathrm{Cr}$.

\section{Experimental}

The EN AA3003 aluminum alloy with a chemical composition shown in Table I was used in this experiment.

The industrially prepared TRC material was cut into samples with dimensions $60 \mathrm{~mm}$ (RD), $80 \mathrm{~mm}$ (TD) and $7 \mathrm{~mm}$ (ND) by a diamond saw. These samples were deformed by the CGP method with die grooves parallel to the rolling direction. The width of grooves is $7 \mathrm{~mm}$ (Fig. 1). Up to three CGP cycles were applied for the specimen preparation. One CGP cycle consist of 4 steps - 2 corrugating and 2 straightening which can together apply effective strain of 1.16 , according to the analytical formula [13].

Composition of the studied material [wt.\%]

TABLE I

\begin{tabular}{c|c|c|c|c|c|c}
\hline \hline $\mathrm{Mn}$ & $\mathrm{Fe}$ & $\mathrm{Si}$ & $\mathrm{Cu}$ & $\mathrm{Zr}$ & $\mathrm{Cr}$ & $\mathrm{Al}$ \\
\hline 1.0 & 0.2 & 0.5 & 0.2 & 0.2 & $\sim 0.1$ & balance
\end{tabular}
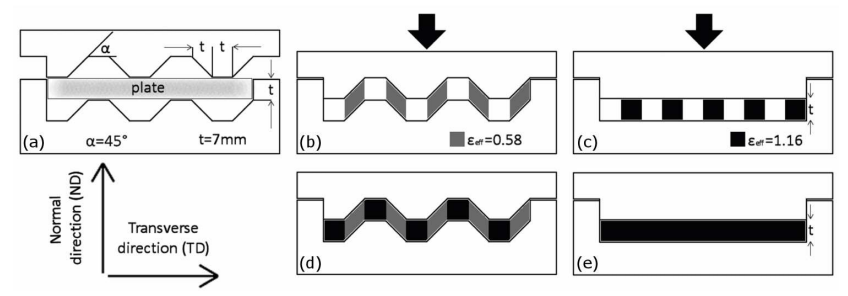

Fig. 1. Scheme of one CGP cycle geometry (a), corrugating (b), (d) and flattening (c), (e).

Samples were polished by $\mathrm{SiC}$ papers and diamond suspensions up to $1 \mu \mathrm{m}$ fineness. Vickers microhardness measurements were done with a load of $100 \mathrm{~g}\left(\mathrm{H} V_{0.1}\right)$. In order to reveal detailed microhardness distribution on cross-section the measurements were done on a large area $20 \times 6 \mathrm{~mm}^{2}$ with a high indent density (around 1500 indents per sample).

Light optical microscopy (LOM) in polarized light was performed on the same samples in order to map the grain structure. These specimens were etched electrolytically with Lectropol 5 in the Barker solution $\left(400 \mathrm{ml} \mathrm{H} \mathrm{H}_{2} \mathrm{O}\right.$ and $12 \mathrm{ml} 35 \% \mathrm{HBO}_{4}$ at $\left.18^{\circ} \mathrm{C}\right)$, and observed with Olympus GX51 microscope. Images were processed by Nis-Elements AR 3.0 software.

Samples for electron backscatter diffraction (EBSD) were polished in the same way as the ones for microhardness measurements, and subsequently electrolytically etched with Lectropol 5 in $33 \% \mathrm{HNO}_{3}$ solution at $-19^{\circ} \mathrm{C}$ and $15 \mathrm{~V}$ for $15 \mathrm{~s}$. The EBSD mapping was carried out in FEI Quanta 200 scanning electron microscope on "harder" (with higher microhardness) and "softer" (with lower microhardness) domains of the cross-section.

Samples for transmission electron microscopy (TEM) were cut and polished to approximately $0.15 \mathrm{~mm}$ thickness and then electrolytically etched with Tenupol 2 in $33 \% \mathrm{HNO}_{3}$ solution at $-19^{\circ} \mathrm{C}$. The TEM observations were done in JEOL JEM 2000FX operated at $200 \mathrm{kV}$.

\section{Results and discussion}

\subsection{Microhardness}

Microhardness measurements of the as-cast material revealed inhomogeneous microhardness distribution. Peripheral areas of the TRC material exhibit higher microhardness as a result of higher cooling rate during casting in the surface areas [2]. Average microhardness of the ascast material is $(54 \pm 1) H V_{0.1}$. A difference of microhardness between surface and central areas is approximately $5 H V_{0.1}$ (Fig. 2).



Fig. 2. Light optical micrograph (left) and microhardness distribution (right) of the as-cast specimen.

The material experienced significant increase of microhardness after the first CGP cycle suppressing the difference between peripheral and central areas (Fig. 3). On the other hand, the microhardness maps revealed alternating areas with higher and lower microhardness corresponding to the groove periodicity of the deformation dies. That is in a qualitative accordance with the finite element method (FEM) simulation of the total equivalent plastic strain as a consequence of slight shifting of the samples during corrugation steps and bending deformation on groove edges (Fig. 3). 


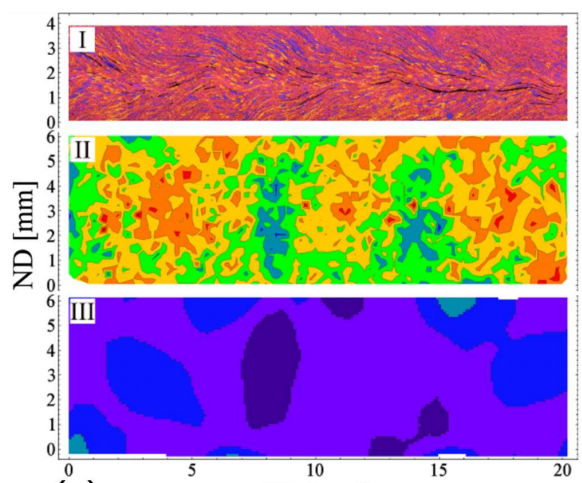

(a)



(b)

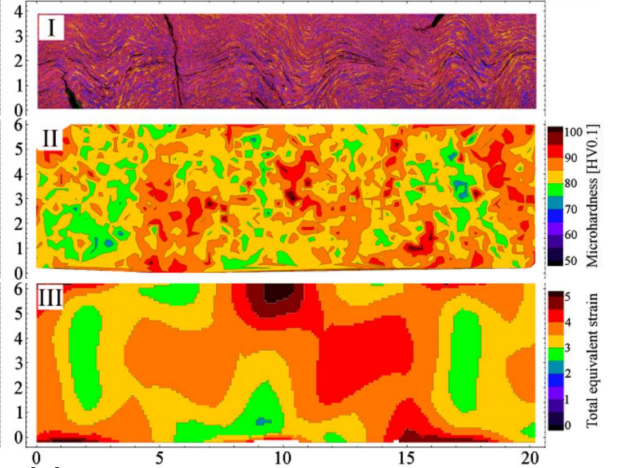

(c)

$\mathrm{TD}[\mathrm{mm}]$

Fig. 3. Light optical micrograph (I) along with microhardness distribution (II) and FEM simulation of total equivalent plastic strain (III) of the specimen after 1 CGP (a), 2 CGP (b), 3 CGP (c).

Subsequent CGP cycles result in saturation of average microhardness (Fig. 4) which almost does not exceed the microhardness reached in the harder areas after 1 CGP cycle (Figs. 3b, c). Domains with a lower microhardness are reduced in favor of domains with higher microhardness. Increase of the average microhardness remains lower than the standard deviation of measurements after the second and third CGP cycle (Fig. 4). This behavior corresponds well to experiments performed on aluminum processed by ECAP, ARB or CGP $[3,11,12,14]$ where saturation was also observed.

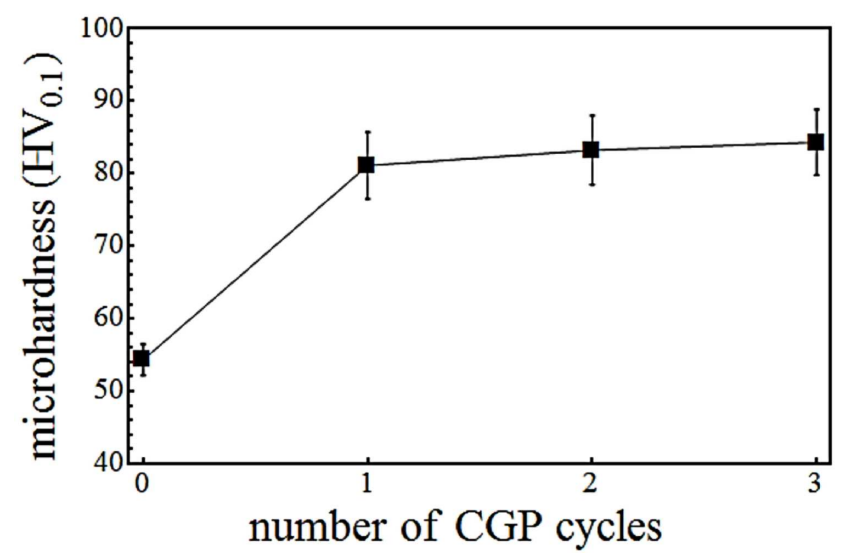

Fig. 4. The influence of the number of CGP passes on microhardness.

\subsection{Light optical microscopy}

A periphery of the as-cast strip contains finer and more flattened grains than the central area (Fig. 2) exhibiting higher hardness in accordance with Hall-Petch relation.

After the CGP deformation the difference between surface and central areas is suppressed (Fig. 3). A grain size (respective grain volume) remains approximately unchanged. Originally parallel flattened grains in the ascast state are bent and turned to varying directions during CGP creating waves which reflect the periodicity of the groove dies. Central segregation is spread by deformation and forms black wavy discontinuous lines in the center of samples (Fig. 3). Formation of cracks during the second and third CGP cycle, displayed as black lines extending from the surface, makes further deformation of this material at room temperature unattainable.

\subsection{TEM and $E B S D$}

TEM of the as-cast sample shows the material containing a relatively low dislocation density (Fig. 5) with dislocations arranged in dislocation walls as commonly observed in TRC alloys [15].
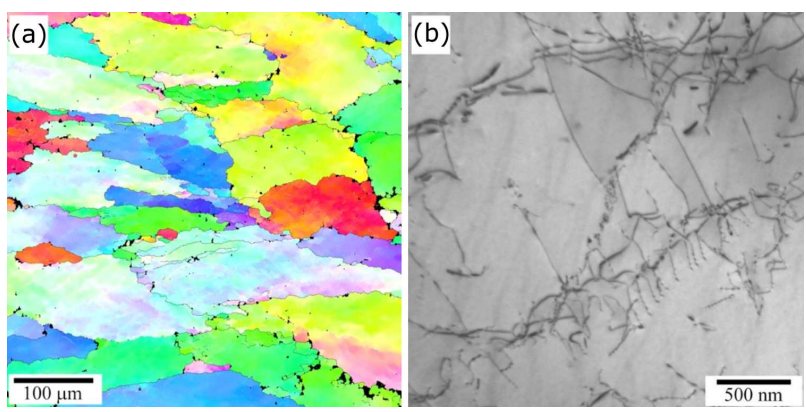

Fig. 5. Microstructure of the as-cast material, the EBSD orientation maps (a) and TEM image (b).

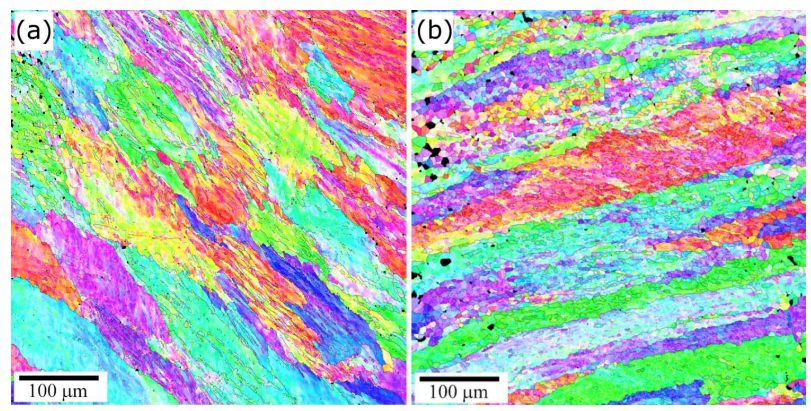

Fig. 6. The EBSD orientation maps: after 3 CGP, softer area (a) and harder area (b). 


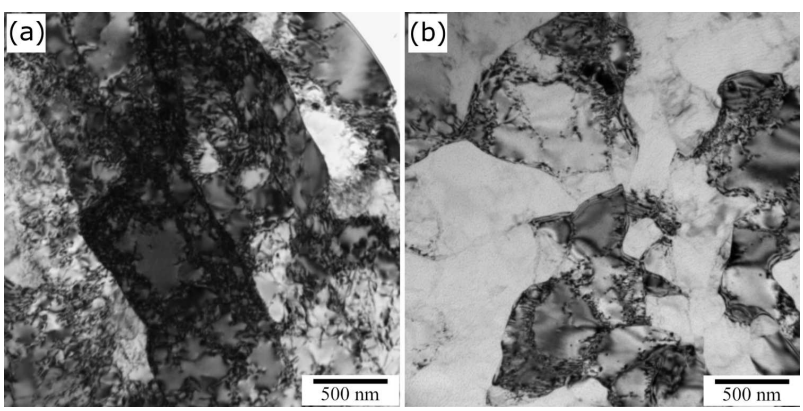

Fig. 7. The microstructure: after 3 CGP, softer area (a) and harder area (b).

CGP leads to a significant hardening. Calculated inhomogeneity of the total equivalent plastic strain distribution is replicated by a difference in microstructure of domains with higher microhardness and domains with lower microhardness. The EBSD orientation map exhibits a pronounced fragmentation into subgrains in the harder domains of the sample after 3 CGP cycles (Fig. 6b). TEM revealed a well-defined subgrain structure (Fig. 7). Surprisingly, domains with lower microhardness have a distinctly higher dislocation density (Fig. 7a) while harder domains exhibit higher degree of recovery (Fig. $7 \mathrm{~b}$ ).

The observed anomalous behavior of microhardness is in a good agreement with recent works on SPD materials $[11-13,16]$ and is related to dynamic recovery and polygonization due to the adiabatic heating emerging during deformation $[13,14,16]$.

The most probable explanation of this phenomenon is that free dislocations as well as dislocation sources present in SPD processed materials make the yielding relatively easier than yielding of the material with recovered subgrains where it is difficult to activate and multiply dislocations within the small grains or subgrains $[6,7,9$ 12]. As a result higher hardness was observed in the material with the higher accumulated deformation energy and higher degree of recovery.

\section{Summary}

CGP forms alternating domains with higher and lower microhardness in the EN AA 3003 TRC aluminum alloy. Their distribution is in a qualitative accordance with the FEM simulation of the total equivalent plastic strain but unlike the simulation microhardness saturates due to the dynamic recovery confirmed by TEM observation and EBSD measurements. Unexpectedly, harder domains, also with higher accumulated deformation, have a lower dislocation density. The most probable explanation is that a material with a lack of free dislocations and dislocation sources requires more energy to activate and multiply dislocations within the small grains or subgrains which makes deformation of the harder domains more difficult.

\section{Acknowledgments}

The financial support of grant GAUK 134715 is gratefully acknowledged. The presented work was financially supported by the Ministry of Education, Youth and Sport Czech Republic — project LQ1603 Research for SUSEN. This work has been realized within the SUSEN Project, established in the framework of the European Regional Development Fund (ERDF) in project CZ. 1.05/2.1.00/03.0108 and of the European Strategy Forum on Research Infrastructures (ESFRI) in the project CZ.02.1.01/0.0/0.0/15 008/0000293, which is financially supported by the Ministry of Education, Youth and Sports.

\section{References}

[1] L. Huang, G. Huang, L. Cao, X. Wu, Z. Jia, M. Xia, Q. Liu, Mater. Sci. Eng. A 682, 63 (2016).

[2] N.S. Barekar, S. Das, X. Yang, Y. Huang, O. El Fakir, A.G. Bhagurkar, L. Zhou, Z. Fan, Mater. Sci. Eng. A 650, 365 (2016).

[3] T. Koizumi, M. Kuroda, Mater. Sci. Eng. A 710, 300 (2018).

[4] E.O. Hall, Proc. Phys. Soc. London Sect. B 64, 747 (1951).

[5] G.I. Taylor, F. R. S., Proc. R. Soc. London Ser. A 145, 362 (Part I) (1934), ibid (Part II).

[6] N. Tsuji, Y. Ito, Y. Saito, Y. Minamino, Scr. Mater. 47, 893 (2002).

[7] N. Kamikawa, X. Huang, N. Tsuji, N. Hansen, Acta Mater. 57, 4198 (2009).

[8] J. Gubicza, N.Q. Chinh, Gy. Krállics, I. Schiller, T. Ungár, Curr. Appl Phys. 6, 194 (2006).

[9] D. Terada, H. Houda, N. Tsuji, J. Mater. Sci. 43, 7331 (2008).

[10] M. Šlapáková Poková, M. Zimina, M. Cieslar, Trans. Nonferrous Met. Soc. China, 26, 627 (2016).

[11] F. Khakbaz, M. Kazeminezhad, J. Manuf. Process. 14, 20 (2012).

[12] J. Zrnik, T. Kovarik, Z. Novy, M. Cieslar, Mater. Sci. Eng. A 503, 126 (2009).

[13] F. Khodabakhshi, M. Haghshenas, H. Eskandari, B. Koohbor, Mater. Sci. Eng. A 636, 331 (2015).

[14] Y. Miyajima, M. Mitsuhara, S. Hata, H. Nakashima, N. Tsuji, Mater. Sci. Eng. A 528, 776 (2010).

[15] M. Poková, M. Cieslar, J. Lacaze, Acta Phys. Pol. A 122, 625 (2012).

[16] S.S. Satheesh Kumar, T. Raghu, Mater. Des. 57, 114 (2014). 